\author{
Military Technical College \\ Kobry El-Kobbah, \\ Cairo, Egypt
}

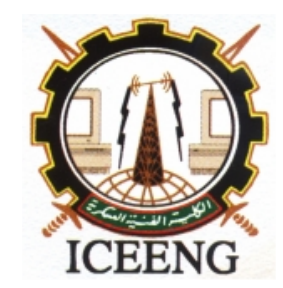

\title{
Improvement of UWB - multiband OFDM BER performance using Barker signaling
}

\section{$7^{\text {th }}$ International Conference on Electrical Engineering \\ ICEENG 2010}

\author{
By \\ Abdel-Rahman Al-Qawasmi *
}

$\underline{\text { Abstract: }}$

Orthogonal Frequency Division Multiplexing (OFDM) technology is a key technique for achieving the high data rate and spectral efficiency requirements for wireless communication systems of the near future. UWB - Multiband OFDM has an acceptable BER performance. In a multipath noisy channel the bit error is very high. In this Paper we present the Barker code as a coding technique and solution to increase the BER performance using the correlation detection. The system performance is increased up to $11 \mathrm{~dB}$ for autocorrelation detection. The drawbacks of the system are the additional required bandwidth, congestion of the channel and the unexpected error position in the Barker sequence. In order to minimize bandwidth, Time-Frequency-Time using Direct and reversed Fourier transforms is used based on acoustic electronic processors.

\section{Keywords:}

OFDM, Barker sequence, BER

* Philadelphia University, Jordan

** Mutah University, Jordan 


\section{Introduction:}

OFDM is a sub-class of multicarrier modulation (MCM) that combines parallel data transmission with frequency division multiplexing (FDM) technique and allows spectral overlap of subchannels. The idea is to transmit single high-rate data stream over multiple parallel low-rate data streams [3].

Modern digital radio should be a high speed and has the reliability of transmitting data under different conditions of signals propagation with minimum error rate. Technology of OFDM provides high spectrum efficiency and has the ability to cope with distortions of signals caused by channel of communication with Multipath propagation. The high data rate in OFDM systems is achieved by the parallel transmission of information over a significant numbers of orthogonal sub channels [1].

Adaptive modulation is only suitable for duplex communication between two stations, since the transmission parameters have to be adapted using some form of two-way transmission in order to allow channel measurements and signalling to take place. In order to efficiently react to the changes in channel quality, we need to have channel quality estimation, choose the appropriate parameters for the next transmission and to signal or blind detection of the employed parameters [2].

The OFDM signal is generated using Fast-Fourier Transform (FFT) algorithm. For protection from Inter symbol Interference (ISI), a special save interval is used. To exchange the information over Wireless Local Area Network (WLAN), recently, the standard IEEE 802.11a is used to send data at data rate up to $54 \mathrm{Mbps}$ over area with radius of dozen of meters. Each packet contains information data and preamble for synchronization and channel estimation. QPSK is used for modulation.

Discrete signals with excellent autocorrelation function are appeared in 1950s and 1960s by insensitive researches by specialists in theoretical radio- technique and applied mathematics. These signals have $N$ (Number of positions or bits in barker Code) energy of autocorrelation function and the value of each autocorrelation position equals to unit. Barker codes used in spread spectrum systems without CDMA, that is, without the need for separating different users. A Barker code of length $N=11$ is used in Wireless LANs according to the standard IEEE 802.11 [4].

Barker sequences consists of symbols $a_{n}= \pm 1$ and have autocorrelation functions like

$$
R_{m}=\left\{\begin{array}{ccc}
1 & \text { for } & m=0 \\
0 & \text { for } & m=2 l+1 \\
\pm 1 / N & \text { for } & 2 l
\end{array}\right.
$$

where $l=0,1, \Lambda, \frac{N-1}{2}$. 
The sign in the third line in equation (1) depends on the value of $N$. There are several well-known Barker codes where $N=3,4,5,7,11$ or 13 [5]. In this work Barker code with length $N=11$ is used which has a acceptable main peak ratio with less used energy comparing to Barker code with 13 elements. Figure $1(a, b)$ shows the pulse sequence of Barker Code with 11 elements and its right side autocorrelation function.
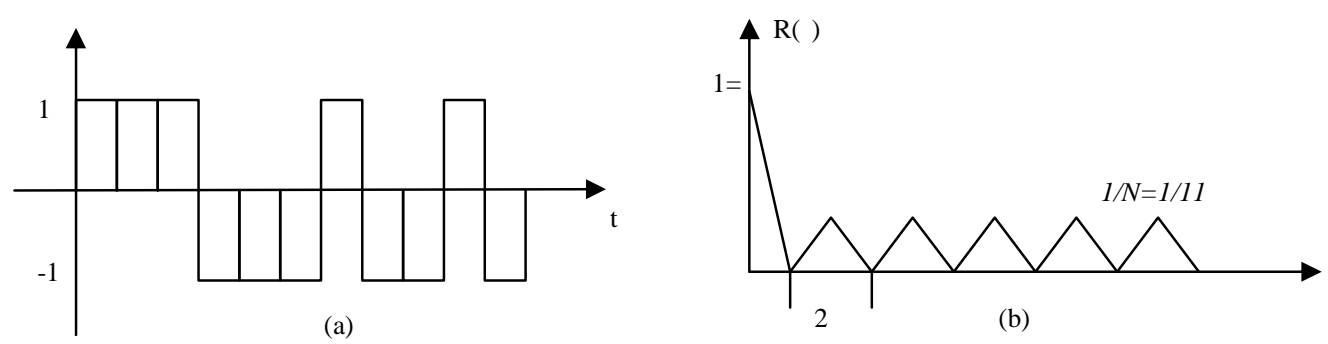

Figure (1): Barker Code (N=11) and its autocorrelation function

It is clearly shown from the Figure 1(b) that the autocorrelation function improves the Signal to Noise Ratio (SNR) by $N$ comparing to secondary peaks.

In this work, we try to replace the ordinary bipolar signaling by Barker code in order to maximize the SNR and at the same time to minimize the Bit Error Rate (BER). The use of OFDM in such BPSK system is required for more efficiently use of the available bandwidth. The amplitude spectrum $|H(x)|$ of code sequence an be found through the energetic spectrum [5]

$$
\begin{aligned}
& |H(x)|^{2}=N\left(1+\frac{1}{N}-\frac{1}{N} \frac{\sin N_{x}}{\sin x}\right) \text { for }-1 / \mathrm{N} \\
& |H(x)|^{2}=N\left(1-\frac{1}{N}-\frac{1}{N} \frac{\sin N_{x}}{\sin x}\right) \text { for }+1 / \mathrm{N}
\end{aligned}
$$

Figure 2 shows the amplitude spectrum for barker sequence using equation (2) 


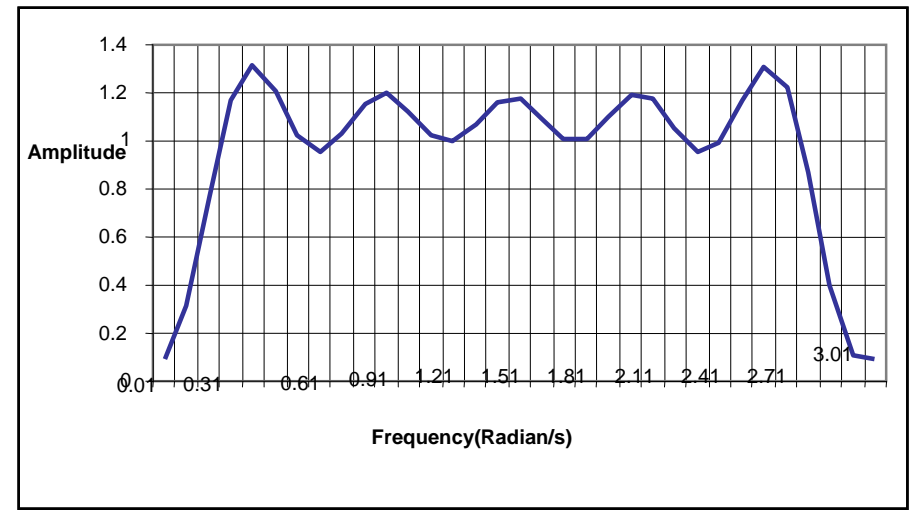

Figure (2): The amplitude spectrum for Barker sequence with $N=11$

If $\omega=[0, \pi]$, the amplitude spectrum has a minimum value that equals to $1 / \sqrt{11}$.

Figure 3 shows $|R(\tau, \Omega)|$ for $N=11$. Where $\Omega$ represents the change in frequency due to Doppler Effect. This is an additional important feature to show and minimize the multipath effect.

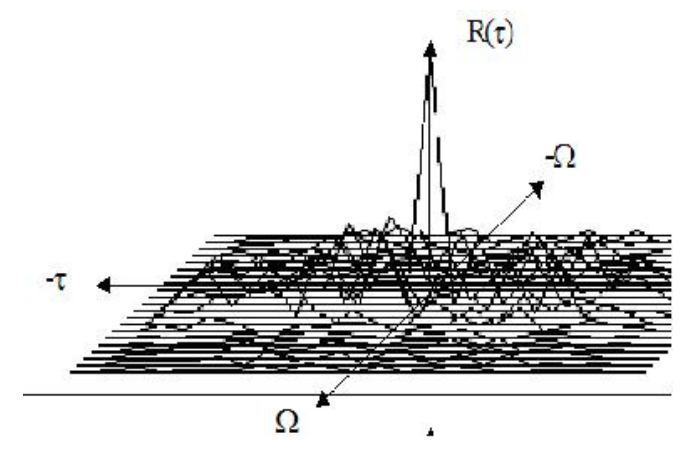

Figure (3): $|R(\tau, \Omega)|$

In this work the Barker code will replace the binary digits in OFDM digital communication in order to make decision more accurate.

A matched filter can be used to detect Barker code. Figure 4 shows schematic structure of a matched filter to detect $N$ positions of Barker code with phase coding. Such code has sequence parts of harmonic frequencies with phase shifts equal to $0^{\circ}$ and $180^{\circ}$. 


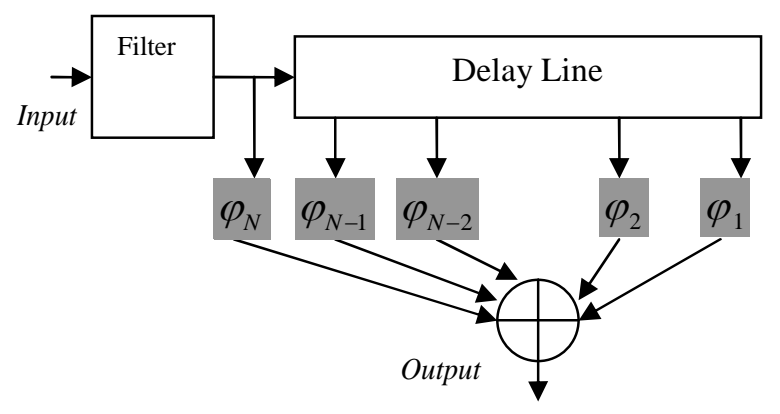

Figure (4): Schematic structure of matched filter

At the input we have a filter that matched with one position of phase modulated signal which has delta-pulse form. At the output of this filter we have radio pulse with rectangular shape form. This pulse is then applied to a delay line with time delay $T$ for every position of signal.

In order to correctly detect the received signal, it is important that the sequence of phase shifts be at the same position of Barker sequence but in the revised order [6]. Figure 5 shows the amplitude spectrum of OFDM Barker signal

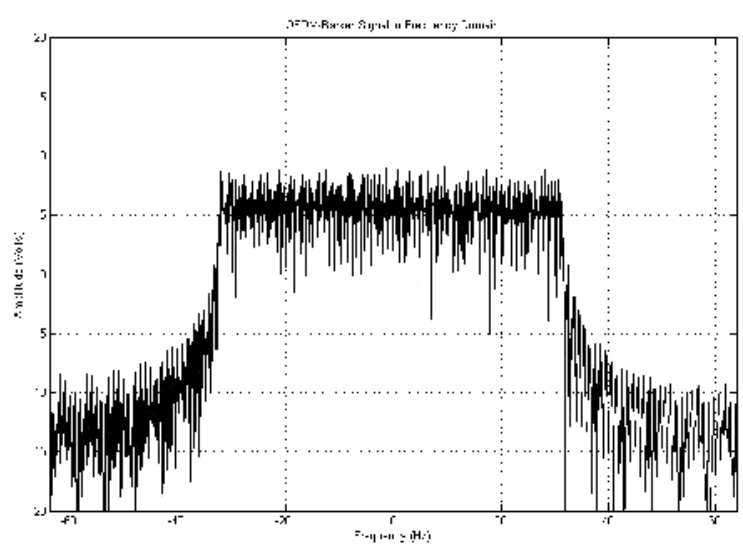

Figure (5): The amplitude spectrum of OFDM Barker signal

\section{OFDM-Barker system model:}

As shown in figure 6, the system model consists of three main stages; Transmitter: in this stage the binary Barker encoded sequences are transmitted using multiband OFDM transmitter; Channel: as the second stage it defined by four different models (CM1, CM2, CM3 and CM4) plus the AWGN that presents in all models. So the channel is noisy and suffering from multipath at the same time; Correlator: as second detection 
stage.

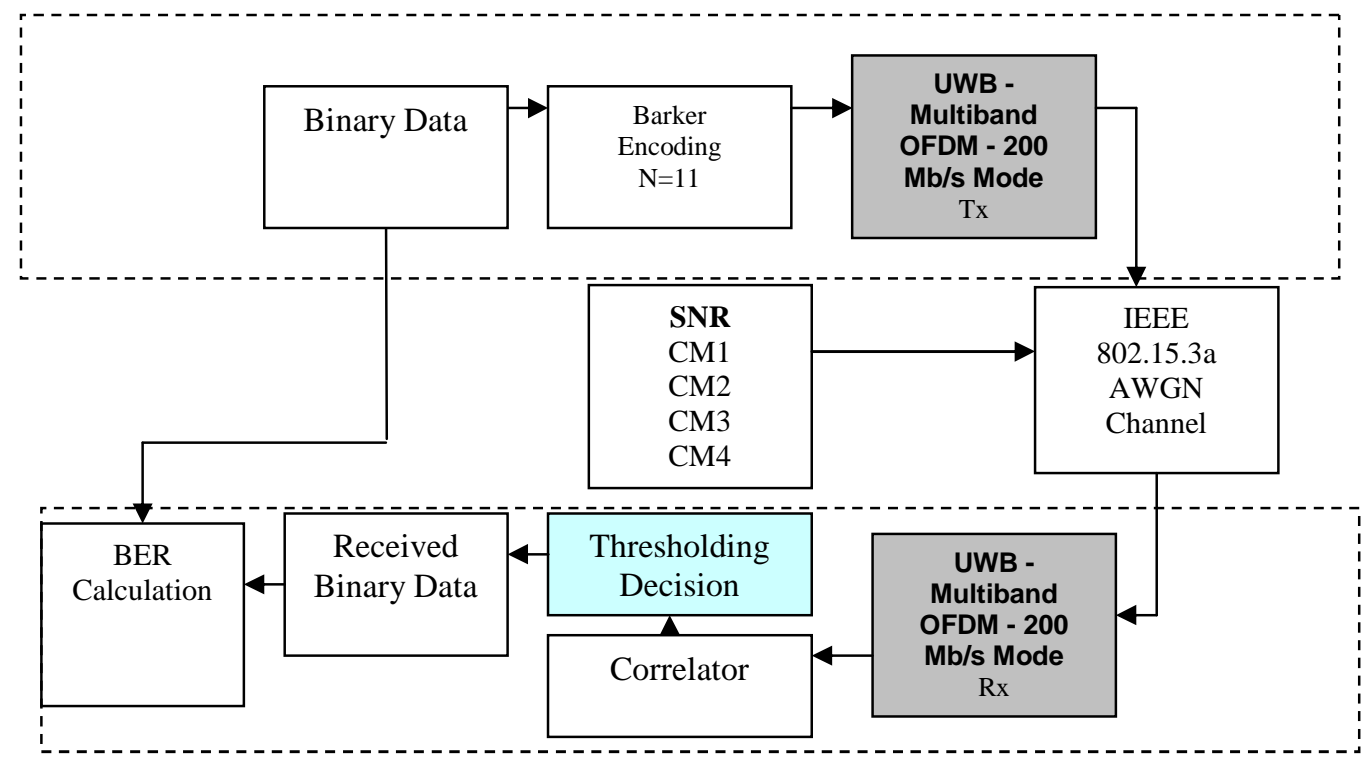

Figure (6): Block diagram of UWB - Multiband OFDM Barker code based system In this work the effect of added AWGN with four types of channels is studied and analyzed. Barker code with 11 elements is used. UWB - Multiband OFDM - $200 \mathrm{Mb} / \mathrm{s}$ Mode is used to transmit and receive data with four different elements as shown in table1. The correlator is added as a second detection process followed by a thresholding decision device to improve the BER performance. In order to make the system more practical, a hard threshold decision is used.

$\gamma=\frac{2\left[R(0)-R_{\max }(\tau)\right]}{R(0)}$

Where $\tau=-11,-10, \ldots, 11$ and $\tau \neq 0$. For $N=11$, the normalized autocorrelation function of received Barker signal has always maximum when $m=0$

$\gamma_{\text {auto }}=2-R_{\max }(\tau)$

The UWB-Multiband OFDM is used with constant IEEE.802.15.3a parameters. The proposed model suffers from the increased number of transmitted binary data which caused increasing in the band width and energy. 
Proceedings of the $7^{\text {th }}$ ICEENG Conference, 25-27 May, 2010

\section{Simulation results and discussion:}

Table (1): UWB-OFDM channels modes

\begin{tabular}{|c|c|c|}
\hline Model & Description & $\begin{array}{c}\text { Distance, } \\
\mathrm{m}\end{array}$ \\
\hline CM1 & Line of sight LoS & $0-4$ \\
\hline CM2 & Non-line of sight NLoS & $0-4$ \\
\hline CM3 & Non-line of sight & $4-10$ \\
\hline CM4 & Non-line of sight & extreme \\
\hline
\end{tabular}

Matlab UWB-OFDM simulation program for transmission and VB for correlation detection are used to extract the results shown in figures 7, 8, 9 and 10. It is clearly seen that the behavior of Barker carves are not standard (not water-flow shape) due to the unpredicted errors in the sequence of received 11 binary digits at the receiver. Figure 7 shows better performance in $8 \mathrm{~dB}$ when $B E R=10^{-4}$ using cross-correlator at the receiving stage and about $11.5 \mathrm{~dB}$ for autocorrelation.

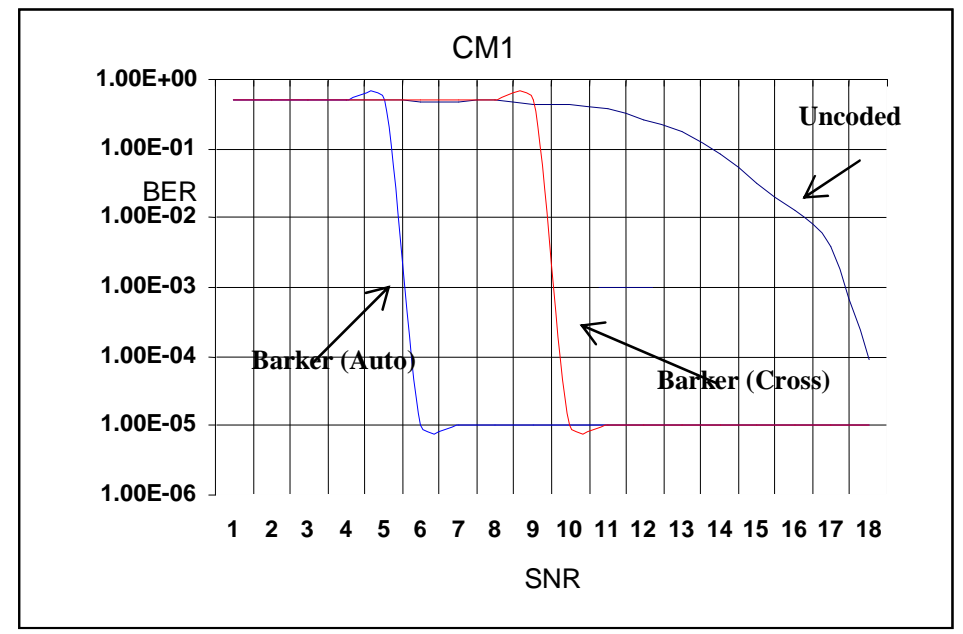

Figure (7): BER performance of UWB OFDM Barker based over LoS CMI

If we represent the binary data using Barker code with 11 elements then the channel information capacity will be decreased. Increasing the data rate will cause increasing in the bandwidth and this will be acceptable due to the ultra wideband system used. In figure 8 the system has better performance when autocorrelation is used. 


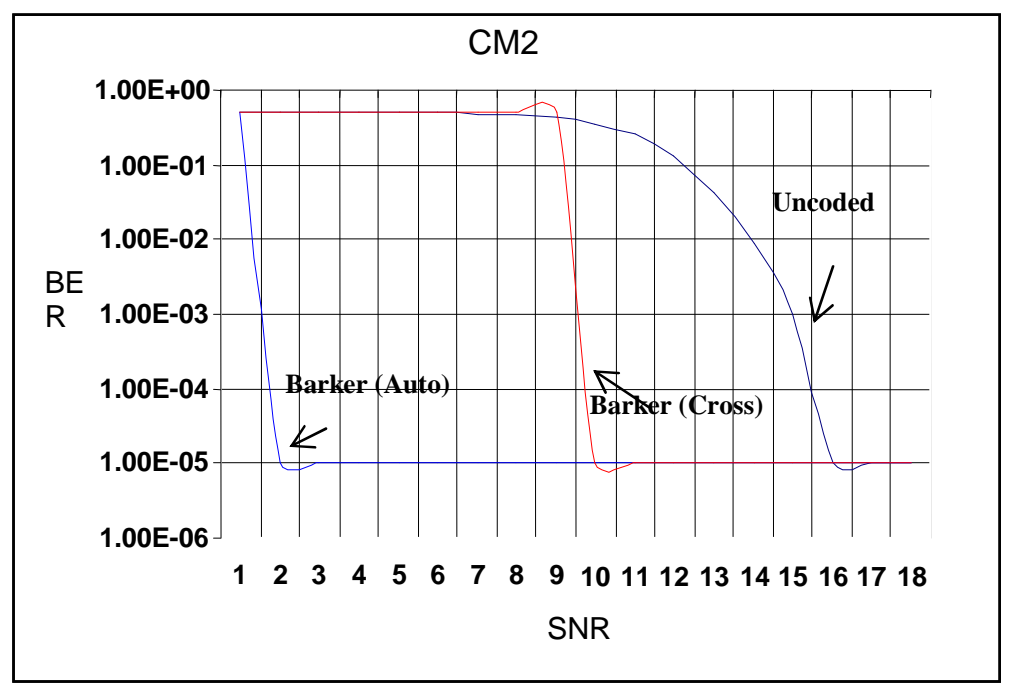

Figure (8): BER performance of Barker based UWB OFDM over NLoS CM2

Practically, the use of autocorrelation is less complex and the cost is minimum comparing with the cross-correlation detection (see figure 11). Also, the autocorrelation function is symmetrical around the Y-axis which provides better detection when $m=0$.

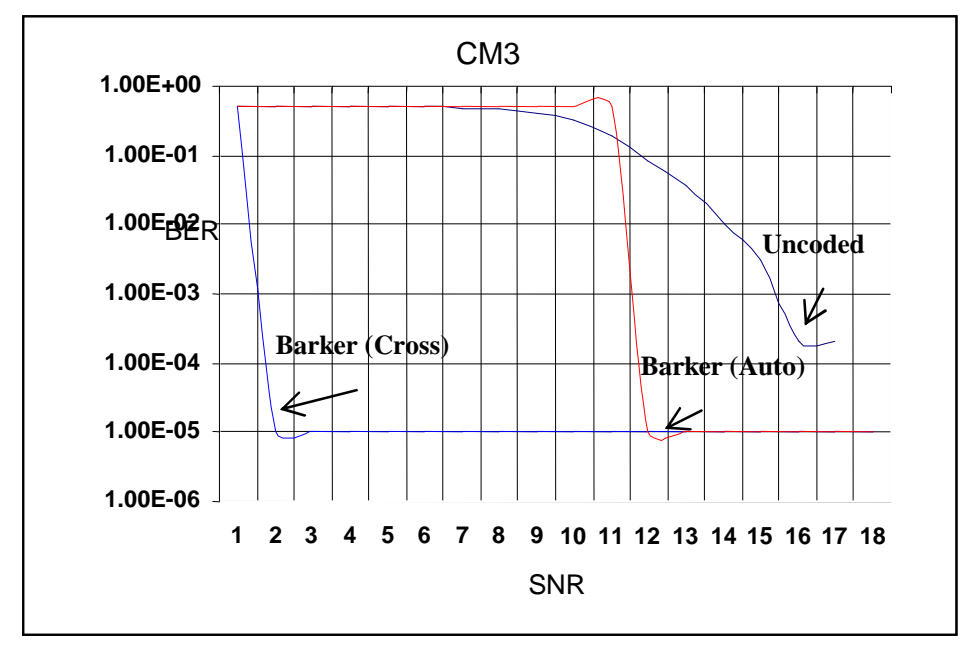

Figure (9): BER performance of Barker based UWB OFDM over NLoS CM3 


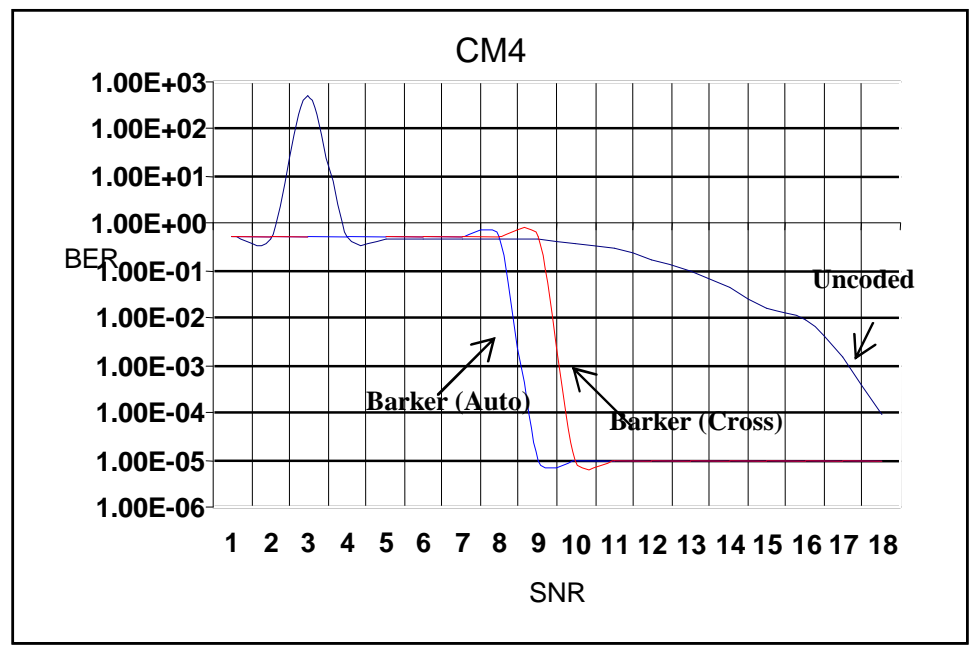

Figure (10): BER performance of Barker based UWB OFDM over NLoS CM4

Depending on the type of channel used, different auto and cross correlations are resulted. In figure 10, the uncoded signal has better performance than the Barker coded signal when the SNR less than 7. But, by increasing the SNR over 7, the Barker coded signal has better performance.

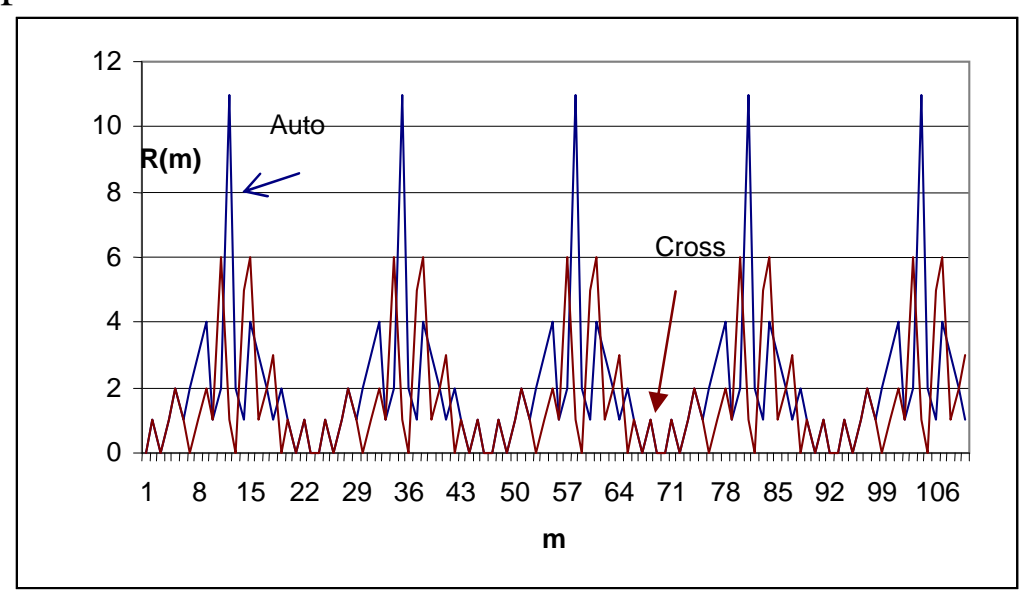

Figure (11): Autocorrelation and cross-correlation functions of received barker coded signal over CM1

\section{Conclusions:}

The BER performance has been analyzed for UWB OFDM Barker based over different channels with AWGN. From the obtained results, it's clearly shown that using Barker code $(\mathrm{N}=11)$ gives better performance for the UWB OFDM systems. Simulated Matlab and VB programs have been built to verify the simulation results as shown in section 3 . 
By Changing the correlation type (auto or cross), it has seen that the autocorrelation detection gives better performance. The decision that used is very hard but if the requirement of the system performance decreased then softer threshold level for decision can be used and more performance can be achieved.

Because the error in UWB OFDM system has a monotone behavior, the cross correlation function has a replica of each other. This can cause a bad effect on the correlation detection process. In other words, the correlation characteristic will be better or worse depending only on detection. In order to avoid this problem, the channel parameters and the effect of AWGN should be made absolutely random.

\section{References:}

[1] L. Hanzo, W. Webb and T. Keller, Single- and multicarrier quadrature amplitude modulation - Principles and applications for personal communications, WLANs and broadcasting, John Wiley \& Sons, Ltd, 2000.

[2] G.R. Lee and J.H. Wen, "The performance of sub-carrier allocation scheme combined with error control coding over OFDM systems," Proc. of NST2002, Dec. 2002.

[3] Prasad R., van Nee R. OFDM Wireless Multimedia Communications. L.: Artech House, 2000. $275 \mathrm{p}$

[4] Henrik Schulze and Christian L"uders "Theory and Applications of OFDM and CDMA", John Wiley \& Sons Ltd, The Atrium, Southern Gate, Chichester, West Sussex PO19 8SQ, England.

[5] L.E. Varakin " Theory of complex signals" Soviet radio, 1970.

[6] Jackson, "Barker R.H., Group synchronizing of binary digital system", Communication theory, London, 1953, P.273 